$$
\begin{aligned}
& \text { ANL/MSD/CP- } 83381 \\
& \text { CONF- } 941144--182
\end{aligned}
$$

\title{
PRODUCTION OF DEFECTS IN METALS BY COLLISION CASCADES: TEM EXPERIMENTS*
}

\author{
M. A. Kirk \\ Materials Science Division \\ Argonne National Laboratory \\ Argonne, Illinois 60439
}

January 1996

The submitted manuscript has been authored by a
contractor of the U.S. Government under contract
No. W-3t-109-ENG-38. Accor dingly, the U.S.
Covernment retains a nonexclusive, royalty-free
license to publish or reproduce the published form
of this contribution, or allow others to do 80 , for

\section{DISCLAIMER}

\begin{abstract}
This report was prepared as an account of work sponsored by an agency of the United States Government. Neither the United States Government nor any agency thereof, nor any of their employees, makes any warranty, express or implied, or assumes any legal liability or responsibility for the accuracy, completeness, or usefulness of any information, apparatus, product, or process disclosed, or represents that its use would not infringe privately owned rights. Reference herein to any specific commercial product, process, or service by trade name, trademark, manufacturer, or otherwise does not necessarily constitute or imply its endorsement, recommendation, or favoring by the United States Government or any agency thereof. The views and opinions of authors expressed herein do not necessarily state or reflect those of the United States Government or any agency thereof.
\end{abstract}

INVITED review paper for the Materials Research Society Meeting, Boston, Massachusetts, November 28 - December 2, 1994.

*Work supported by the U. S. Department of Energy, BES-Materials Sciences, under Contract W-31-109-Eng-38. 


\section{DISCLAMMER}

Portions of this document may be illegible in electronic image products. Images are produced from the best available original document. 


\title{
PRODUCTION OF DEFECTS IN METALS BY COLLISION CASCADES: TEM EXPERIMENTS
}

M. A. Kirk

Argonne National Laboratory, Argonne, IL 60439

\begin{abstract}
I will review our experimental TEM data on the production of dislocation loops by low energy ion bombardment to low doses, as simulations of similar collision cascades produced by fast neutron irradiation, in various metals and alloys. The dependence of vacancy dislocation loop formation on recoil energy, sample temperature, and specific metal or alloy will be examined. Special emphasis will be placed on the effects of dilute alloy additions. A model for cascade melting will be employed to understand these effects, and will require an examination of the role of electron-phonon coupling in cascade cooling and recrystallization. The formation of interstitial dislocation loops as cascade defects, and the influence of the nearby surfaces in these experiments will be briefly discussed.
\end{abstract}

\section{INTRODUCTION}

In this brief review my intention is to summarize the main results of work performed by the author and collaborators over about the last ten years in the field of defect formation by collision cascades in metals. During this period we have made extensive use of transmission electron microscopy (TEM) techniques, often employing in situ ion irradiations in the High Voltage Electron Microscope (HVEM) - Accelerator Facility at Argonne National Laboratory. Also during this period a number of results from computer simulations have emerged which have complimented our experimental results, and taken together, produced what appears to us to be an improved understanding of cascade processes leading to defect formation. Recently, questions concerning defect structure (intersitial or vacancy) ${ }^{1}$ and surface interactions ${ }^{2}$ have arisen which have caused us to reexamine several aspects of the experimental techniques and interpretations. In this regard new experiments are underway and these, with some early results, will also be described.

Interest in cascade damage in metals has existed for many decades in the area of structural and fuel materials used in nuclear reactors. An important result of fast neutron irradiation in these materials is the atomic structural damage created by collision cascades, which result from atomic recoils of several tens of keV energies. Over nearly as long a period of time TEM techniques have provided a useful experimental tool to study the local structure and mechanisms of formation of defects on the size scale appropriate to the collision cascade problem: 1-100 nm. Inspite of this rather long history of experimental work, our understanding of this important damage process is incomplete and has recently been challenged by new experimental, theoretical and computer modeling work. 
In terms of local atomic processes, collision cascades in metals can have numerous results. Over a region roughly the size of a collision cascade $(10 \mathrm{~nm})$ caused by a recoil energy that might be typical of fast neutron damage in copper $(50 \mathrm{keV})$, and if one thinks in terms of a high concentration of atomic defects, interstitials and vacancies, three processes have been generally understood to occur. Due to the proximity of some interstitials and vacancies, substantial recombination will occur. Due to the separation of some interstitials and vacancies, clustering of like defects will occur. And finally, rather few individual interstitials and vacancies will escape the first two processes and be free to migrate away from the original cascade site. The extent to which these individual processes will occur, and thus define the final nascent defect state, will depend on the host atomic (and electronic) structure, the primary recoiling atomic mass and energy, and the temperature of the irradiated metal. And because the collision cascade process is highly statistical in nature, there will be a broad variation in results within a single irradiation-material condition.

The above is a simplified and conventional description of defect formation by collision cascades, which we now believe to be true only in rather diffuse collision cascades of low energy density. From our experimental results and recent computer modeling results described below, this description must be modified to include local lattice melting.

Molecular dynamic calculations ${ }^{3}$, primarily of collision cascade events of $5-20 \mathrm{keV}$ in $\mathrm{Cu}$ and $\mathrm{Ni}$, have revealed the occurence of a liquid-like state, or melting, subsequent to the collisional phase $\left(10^{-13} \mathrm{sec}\right)$ and covering the cooling of a thermal spike phase (up to $10^{-11} \mathrm{sec}$ ). These results immediately suggested to us a mechanism for the formation of a vacancy dislocation loop under such conditions ${ }^{4}$. Surviving interstitials are found outside the melted zone. The rate at which the melted zone recrystallizes will determine the spatial distribution of the remaining vacancies. A fast recrystallization rate will tend to leave vacancies more dispersed, thus reducing the probability of loop formation. However, a slow recrystallization rate will allow a more perfect recrystallization front to move inwards and, in effect, sweep the remaining vacancies towards the center of the melt zone, thus enhancing the probability of loop formation. This mechanism will naturally explain the experimental results to be described: the dependence of loop formation on lattice temperature and cascade energy density, the results of cascade overlap, and a dependence on different metals (melting temperature) to some extent. Also, in ordered alloys the dependence of disordered zone sizes on cascade energy density and irradiation temperature, as will be shown here, are easily explained by a strong dependence of disordering on the formation and size of a melted volume within the thermal spike phase.

Experiments using TEM can only generally "see" the end result of the above cascade process, and then only if it includes a cluster of point defects of sufficient size ( $>1 \mathrm{~nm}$ under the best imaging conditions, but more often $>1.5 \mathrm{~nm}$ under common imaging conditions), which also produces a strain field and/or stacking fault used to image under diffraction contrast conditions. Such defects in metals are dislocation loops and stacking fault tetrahedra, which can be imaged individually at lower irradiation doses, typically $<10^{18}$ neutrons $/ \mathrm{cm}^{2}\left(E_{n}>0.1 \mathrm{MeV}\right),<10^{12} \mathrm{Kr}+(1 \mathrm{MeV}) / \mathrm{cm}^{2}$, or $<10^{16}$ protons $(3 \mathrm{MeV}) / \mathrm{cm}^{2}$, as examples. At higher doses complex dislocation arrangements can result, usually a product of dislocation climb and interactions. The development of 
complex dislocation and voided microstructures will be determined by biased sink reactions between freely migrating point defects or small clusters and existing dislocations produced by previous material processing or individual cascades. The point to be made here is that this development of microstructure at high irradiation doses depends strongly on the formation of dislocation loops and stacking fault tetrahedra at individual cascade sites. These cascade defects will provide biased sinks and sources for migrating point defects, depending sensitively on the material temperature. This leads us to mention a current controversy of whether these individual collision cascades can produce, with substantial probability, interstitial loops ${ }^{1}$. Much previous experimental work has identified the nature of loops produced from collision cascades to be vacancy. Room for some doubt on this identification does exist and will be discussed later in the paper.

Other cascade processes in metals which should be mentioned include anti-site disordering, atomic mixing across a phase boundary, and amorphization. Disordering within individual cascade volumes has been a valuable tool in the study of cascade defect production, and some of our TEM experiments in this area will be reviewed here.

\section{EXPERIMENTAL}

Under both fast neutron (degraded fission or fusion neutron energy spectra) and $\mathrm{MeV}$ ion irradiations, a wide spectrum of atom recoil energies is present in the irradiated material 5 . One could define an average recoil energy based on these distributions, giving equal weight to low and high energy recoils. But if interest lies with total defect production or especially just cascade defects, then a distribution weighted by the recoil energy (often corrected for electronic energy losses which do not contribute to defect production in most metals, and usually termed damage energy) is more useful to illustrate the recoil energy range of importance 6 . Of primary interest in our work has been the study of collision cascades typical for fast neutron irradiations, which can be defined by the median of the damage energy distribution, or about $50 \mathrm{keV}$ recoil energy in copper.

To study the production of dislocation loops and stacking fault tetrahedra in individual collision cascade events, we have used irradiation of metals and alloys of interest by ions with masses ranging from $\mathrm{Ar}$ to $\mathrm{Xe}$ and energies from 40 to $100 \mathrm{keV}$. Thus, a recoil atom under neutron irradiation generally in a bulk material, is simulated by an incident ion, producing a near surface collision cascade event. This is conveniently done and the simulated cascade is contained in a metal sample of thickness appropriate for TEM, 30-100 nm. Details of the physical mechanisms for cascade defect formation can be investigated by varying the ion mass and energy, varying the pure metal or alloy and its irradiation temperature, and varying the ion dose. Of great use in these studies has been the in situ ion irradiation and TEM facility at Argonne.

Standard conventional dark field electron microscopy techniques have been used to produce images of cascade defects. Diffraction conditions almost always have consisted of slight deviations from a strong two-beam Bragg condition. Since our primary interest has been in defect identification, defect sizes and areal density of defects, we have not normally used weak beam conditions due to the frequent 
occurrence of misleading or artifact images. The in situ nature of many experiments has allowed us to study defect formation in exactly the same sample area as a function of ion dose or sample temperature. Reasonably accurate ion dosimetry (10\%) allows a determination of TEM visible defect probability per incident ion or defect yield.

\section{RESULTS and DISCUSSIONS}

\section{Pure Metals: $\mathrm{Cu}, \mathrm{Ni}, \mathrm{Fe}$ and $\mathrm{Al}$}

Our earliest experiments were designed to investigate the temperature and dose dependence of dislocation loop formation in a selected number of pure metals. Collision cascades were produced by both self ion and $\mathrm{Kr}$ ion irradiations over a range of doses. Bombarding ion energies were usually $50 \mathrm{keV}$ and occasionally $100 \mathrm{keV}$. Irradiated sample temperature and in situ TEM was usually performed at either $300 \mathrm{~K}$ or $30 \mathrm{~K}$, the latter sufficiently low to restrict both vacancy and interstitial motion in these metals. Detailed results from these experiments can be found in the original references 4, 7-9 and are reviewed in a recent paper by Jenkins, Kirk and Phythian ${ }^{1}$. A brief summary of conclusions formed from these data include the following:

1. The probabilities of cascade collapse to dislocation loop (or stacking fault tetrahedra in $\mathrm{Cu}$ and $\mathrm{Ni}$ ) are highest for $\mathrm{Cu}(0.5)$, followed in order by $\mathrm{Ni}(0.14)$ and $\mathrm{Fe}(0.0)$, for self ion irradiations of $50 \mathrm{keV}^{8}$ to low doses at $300 \mathrm{~K}$. Defects are produced in Fe only at high doses at both 30 and $300 \mathrm{~K}$, indicating a loop formation mechanism which requires cascade overlap to produce sufficiently high local vacancy concentrations. Defect formation in $\mathrm{Al}$ is less likely than in Ni for comparable irradiation conditions: probabilities of 0.06 and $0.20 \mathrm{~m}$ respectively, for $50 \mathrm{keV} \mathrm{Kr}+$ irradiation at $300 \mathrm{~K}^{7}$.

2. Defect formation occurred at $30 \mathrm{~K}$ with about half the probability of that at $300 \mathrm{~K}$ in $\mathrm{Cu}$ and $\mathrm{Ni} 4,8,10$. That it occurred at all at $30 \mathrm{~K}$ indicates the athermal nature of dislocation loop formation within the lifetime of the cascade process. However, the temperature dependence indicates a role of lattice temperature in the process.

3. Defect density as a function of dose shows a saturation behavior in $\mathrm{Cu}$ and $\mathrm{Ni}$, as it certainly must. The important observation of the saturation mechanism, even at moderate doses, which shows direct evidence of dislocation loop reorientation, coalescence and dissolution, must be explained by the overlap of defect cascades in space 4,10 .

4. The observation of a greater than linear increase in dislocation loop density with dose, in those metals with a very low probability for loop formation at low doses (Fe and Al), is direct evidence for the existence of submicroscopic vacancy clusters, stable at 30 and $300 \mathrm{~K}$, and which do not collapse to form loops until overlapped by a second cascade 7,8 .

We have interpreted all the above results in terms of the formation of vacancy dislocation loops (including some stacking fault tetrahedra) within individual collision cascades, and as representative of behavior in the bulk as by neutron irradiation. This interpretation can be questioned in light of recent neutron irradiation experiments ${ }^{11}$. 
However, earlier results of low dose fission neutron irradiated $\mathrm{Cu} 12$ and dilute $\mathrm{Cu}$ alloys 13 has found in the first case that the dislocation loops are primarily vacancy in nature and at a density to be expected from the calculated concentration of cascade events and ion results for the probability of loop formation. A small concentration were determined to be interstitial, and the sizes and concentrations of these were found to be dependent on the dilute alloy, indicating that they were formed not from cascade events, but by the clustering of mobile interstitials. The TEM method of loop nature determination was a stereo depth measurement and coincident image of the defect strain field (black/white method), a rather difficult method to apply.

Those low dose neutron irradiation experiments which resulted in a determination of interstitial loop nature relied extensively on the so-called $21 / 2 \mathrm{D}$ method ${ }^{11}$. Because of this striking disagreement between results for loop nature in neutron irradiated samples, a recent collaboration was formed ${ }^{14}$ to investigate rather rigorously these two TEM methods, and to determine under what conditions, if any, interstitial loops may be formed directly at collision cascade sites. A resolution of this question is quite important to fundamental defect production mechanisms, which are the basis for theoretical models to predict macroscopic property changes under high dose neutron irradiation. Further prediction of interstitial loop formation has come from recent computer simulations of collision cascade events ${ }^{15}$.

Preliminary results of the collaborative experiments in $\mathrm{Cu}$ now suggest that the 2 $1 / 2 \mathrm{D}$ method for loop nature determination cannot be reliably applied ${ }^{14}$. Whether it can be used under specific restrictive circumstances, if at all, is under current investigation. Important previously published results using this method must certainly be reevaluated carefully. This will be done when our results are final.

There is little doubt that the great majority of dislocation loops produced by low energy ion irradiations are of vacancy nature, as we have measured ourselves and as considerable past work has shown ${ }^{1}$. However, a role of the surface to produce a mechanism for vacancy loop formation has recently been proposed by computer simulation ${ }^{2}$. The observation of vacancy loops following low dose neutron irradiations mentioned above does argue against such a mechanism as necessary to produce such loops.

Under current investigation is the detailed role, any, of the surface in the formation of vacancy dislocation loops. One method being used is careful stereo measurements of disordered zones produced by low energy ion bombardment of ordered alloys ${ }^{16}$. The morphology of individual disordered zones and its relationship to the surface of ion incidence, and the loop formation probability will be determined. Preliminary results in this work reveals a notable lack of disordering near the surface at the position of ion entry. If this results holds in more complete studies, its interpretation suggests an interesting effect of the surface, but one which may not significantly influence the formation probability of vacancy dislocation loops. The use of ordered alloys in other cascade studies has produced informative results as reviewed below. 


\section{Ordered Alloys: $\mathrm{Cu}_{3} \mathrm{Au}_{\mathrm{u}}$ and $\mathrm{Ni}_{3} \mathrm{Al}$}

Several interesting results were produced in experiments by Black et al ${ }^{17}$ in the ordered alloy $\mathrm{Cu}_{3} \mathrm{Au}$. A range of ion masses ( $\mathrm{Ar}, \mathrm{Cu}$ and $\mathrm{Kr}$ ), two bombarding energies (50 and $100 \mathrm{keV}$ ), and two sample temperatures (30 and $300 \mathrm{~K}$ ), were employed to study the relationships between disordered zone sizes and probabilities of dislocation loop formation. As every incident ion produced a disordered zone (imaged using a superlattice reflection) it was possible to "see" each individual cascade event, and discover why some produce a dislocation loop (imaged using a fundamental reflection) and others do not. The answer lay in the rather wide distribution of collision cascade sizes within a given irradiation condition. For a single irradiation condition, it was found that those cascades which produce the largest disordered zones had the greatest probability of loop formation. Thus, within the statistical variations of many collision cascades, and subsequent thermal-like atomic agitation before cooling, one can associate a greater amount of disordering with a higher probability of loop formation.

The analytical theory of cascade sizes ${ }^{18}$ predicts a decrease in collisional volume in $\mathrm{Cu}_{3} \mathrm{Au}$ with increasing bombarding ion mass from $\mathrm{Ar}$ to $\mathrm{Kr}$. The measured size distributions of disordered zones followed this trend for irradiations at $30 \mathrm{~K}$, but trended oppositely at $300 \mathrm{~K}$. The significance of this is the suggestion that the extent of disordering is controlled not just by the collisional volume, but also by a thermal spike component which is temperature dependent.

Finally, and perhaps most importantly for this work, is the result that the loop formation probability increased both with increasing bombarding ion mass and with the higher irradiation temperature. These results are based on using the disordered zone density to measure ion dose, and thus is more accurate than in a pure metal measurement, which is dependent on the sometimes limited accuracy of the ion dosimetry. Again, a thermal spike mechanism must be invoked to explain these results. The higher ion mass results in the greater cascade energy density and thus a stronger thermal-like atomic agitation locally within the cascade. Recrystallization from this meltlike region can then lead to an effective loop formation mechanism as discussed earlier. The temperature dependence suggests that the cooling rate of this local thermal spike also plays a key role in the same mechanism.

A similar set of experiments was recently performed by Bui et al [this volume] in the ordered alloy $\mathrm{Ni}_{3} \mathrm{Al}$. Because the order-disorder temperature is considerably higher in this alloy than in $\mathrm{Cu}_{3} \mathrm{Au}$, and the average mass less, different collisional volumes and thermal spike parameters are investigated. Inspite of these differences, results are quite similar. Again, thermal spike ideas must be used to understand both the disordered zone sizes and trends with ion mass and temperature, and the loop formation probabilities. Similar results imply that cascade disordering and loop formation must be closely related to a very strong thermal spike behavior, in other words, melting and then cooling rates must be sufficiently high to retain disorder but also slow enough to allow vacancy agglomeration during recrystallization. 


\section{Dilute Alloys of $\mathrm{Ni}$}

A study of dilute alloy additions to Ni was undertaken to probe their effects on both the cascade collisional phase and the thermal spike phase of loop formation 19 . Nickel was chosen due to its rather low probability for loop formation under self ion and $\mathrm{Kr}$ ion irradiations, and thus possibly sensitive to small changes in the mechanisms of cascade defect formation. Dilute impurities of both undersized (Si) and oversized (Al) atoms in Ni were selected. A striking sensitivity of loop formation probability to each impurity type was indeed found. For both impurity types at low concentrations of about $0.5 \%$ a strong increase of about $70 \%$ in the probability of loop formation was observed. With additions to $4-7 \%$ of both, this probability decreased to that of pure $\mathrm{Ni}$ or slightly less. Dislocation loop size distributions remain unchanged with all $\mathrm{Al}$ concentrations and the low Si concentration (0.6\%). However, the loop size distribution for $4 \% \mathrm{Si}$ in Ni showed a strong increase in the fraction of larger loops and a decrease in the fraction of smaller loops.

In the case of the most dilute additions, we judged the concentration to be large enough, and relative absolute size and mass differences for both impurities large enough, to interrupt the focused collision sequences, thus increasing slightly, but sufficiently, the cascade energy density in, or adjacent to, a melted region. This should enhance the lifetime of the thermal spike process, slowing the recrystallization from the melt, thus increasing the probability of loop formation as observed.

To understand the reduction in loop yield with further additions of both impurities, it was necessary to consider the wide distribution of cascade sizes for the same irradiation condition. In all cases of cascades of varying size, some of the focused collision and replacement collision sequences will be interrupted. For a diffuse cascade with more interstitials well separated from a melted region, a larger loop might be expected, and with some interstitial trapping by $\mathrm{Si}$ impurity atoms, it might become larger in this alloy due to reduced recombination. For a compact cascade with fewer interstitials relatively close to a melted region, a small loop might be expected. However, the enhancement of this volume by the interruption of focused collision sequences to include even more interstitials will produce an even smaller loop in the same alloy. Thus, large loops become larger in the case of Si additions of $4 \%$, and small loops become smaller in both $4 \%$ Si and $7 \%$ Al alloys. A decrease in observed loop yield is then understood to result from the smaller loops becoming too small to resolve by TEM.

In summary of these dilute alloy results, we interpret them to suggest that both undersized (Si) and oversized (Al) impurity atoms in Ni disrupt focused collision and replacement collision sequences, increasing both melt zone size and slowing recrystallization. These effects combined with consideration of a wide distribution of cascade sizes, and including interstitial trapping by Si, permit an understanding of both average loop yield and size changes with these dilute alloy additions. 
Materials Dependence

A series of non dilute alloy experiments 20 and an assessment of published data 21 have yielded information on how the loop formation probability under cascade producing irradiations depends on the particular metal or alloy. In addition to the above demonstrated dependence on cascade energy density, irradiation temperature, and dilute alloy additions, factors such as melting temperature and stacking fault energy are found to be important in some cases. Perhaps most surprisingly, a dependence on the degree of coupling between the electronic and atomic systems was found to play a significant role in those metals with strong electron-phonon coupling.

The rather low probability of loop formation in Ni compared with $\mathrm{Cu}$, metals with the same structure and similar atomic mass and density, and the virtually zero probability of loop formation by self ions in Fe, suggested to us quite early that a coupling between the atomic energy of motion within the cascade and the electronic system may provide a mechanism of rapid cooling of the thermal spike phase, and thus reduce the probability of loop formation in the defect cascade. Independently, a theoretical treatment of this coupling was published by Flynn and Averback 22 , and subsequently used by us to characterize the strength of this coupling in various metals and alloys.

To experimentally test the idea, a full series of $\mathrm{Cu}-\mathrm{Ni}$ alloys was used to study dislocation loop formation by collision cascades of the same energy density with $50 \mathrm{keV}$ $\mathrm{Kr}$ ion bombardment 20 . The loop yield and total vacancy retention in loops was measured to decrease from pure $\mathrm{Cu}$ to $\mathrm{Ni}$, not in a smooth or linear fashion, but rather rapidly from $40-60 \% \mathrm{Ni}$ in $\mathrm{Cu}$. This was then compared to three possible explanations: an increase of melting temperature with increasing $\mathrm{Ni}$ content, an increase of stacking fault energy with increasing $\mathrm{Ni}$ content, and an increase of power transferred from the atomic to the electronic system with increasing Ni content. All three trends predict a decrease in loop yield with increasing $\mathrm{Ni}$ content, but only the "electron-phonon" coupling shows a sharp increase from $40-60 \% \mathrm{Ni}$, while the melting temperature shows a nearly linear variation over the full alloy range, and the stacking fault energy actually shows a slight decrease in the $40-60 \%$ Ni range, which would predict an increase in yield rather than the decrease observed. Thus the best match of the form of the alloy dependence in the loop yield data is shown by the prediction of coupling between the atomic and electronic systems. A similar analysis of published data in the Ni-Cr system proved even more conclusive for the same mechanism. Published data in several $\mathrm{Cu}$ alloy systems (exclusive of $\mathrm{Ni}$ ) ${ }^{23}$, where the coupling between atomic and electronic systems is calculated to be negligible, show a remarkable consistency of loop yield with melting temperature 20 in these Cu alloys.

To further demonstrate the importance of coupling between the atomic and electronic systems in some elemental metals, a compilation of reliable TEM data on experimentally determined loop yields for 17 different metals under 38 different ion irradiation conditions was constructed 21 . When only those metals with reasonably strong contributions of calculated power transfer to the electronic systems are compared under irradiation conditions which produce similar cascade energy densities, a systematic dependence on the strength of coupling is revealed. The strength of this 
coupling in these metals proceeds from a moderate value for $\mathrm{Ni}$, increases in the order of $\mathrm{Co}, \mathrm{V}, \mathrm{Cr}, \mathrm{Ti}$, with the highest value for $\mathrm{Fe}$. Thus, a thermal spike cooling mechanism based on this coupling is certainly the reason for self ion and neutron irradiations of $\mathrm{Fe}$ to produce no resolvable dislocation loops at isolated cascade sites 9 . A more detailed discussion of this work can be found in the paper by Tappin in this volume.

\section{SUMMARY}

A review of our TEM experiments on factors affecting the probability of dislocation loop formation in defect cascades is summarized in the following main points.

1. Cascade energy density is a strong factor in the production of vacancy dislocation loops by defect cascades. Generally, the higher the cascade energy density in a given metal, the higher the probability for loop formation. A low cascade energy density for self ion and neutron irradiation of Aluminum is believed to be the central reason for a very low probability of loop formation.

2. The sample temperature during irradiation influences the probability of loop formation. The probability increases by about a factor of 2 from 30 to $300 \mathrm{~K}$ in $\mathrm{Cu}, \mathrm{Ni}$ and $\mathrm{Cu}_{3} \mathrm{Au}$.

3. At higher doses the overlap in space of two cascade events can produce an increased probability for loop formation in a metal with small loop yield at low doses, and a decreased probability in a metal with a larger loop yield at low doses (saturation effect).

4. Dilute alloys show complex behavior of loop formation probability dictated by interruption of focused collision sequences during the collisional phase and reduction of recombination due to trapping of interstitials during the thermal spike phase.

5. A reduction in the probability of loop formation by rapid cooling of the thermal spike phase resulting from a strong coupling of atomic and electronic systems is demonstrated in $\mathrm{Ni}, \mathrm{Co}, \mathrm{V}, \mathrm{Cr}, \mathrm{Ti}$ and especially $\mathrm{Fe}$.

6. All of the above can be explained within a model of thermal spike melting and recrystallization during the cascade lifetime $\left(10^{-11} \mathrm{sec}\right)$, which has been suggested by computer simulations of cascade events.

Under current investigations are a rigorous experimental search for evidence of interstitial loops formed at cascade events, the influence of a nearby surface on the probability of vacancy loop formation, and an evaluation of two existing TEM methods to determine loop nature (vacancy or interstitial). Preliminary results indicate that the black/white method, although difficult to apply, is more reliable than the so-called $21 / 2$ D method. 


\section{ACKNOWLEDGMENTS}

The author is grateful for financial support from the U.S. Department of Energy, Office of Basic Energy Sciences-Materials Science under contract No. W-31-109-Eng38. Most of this work has been done in collaboration with Prof. lan Robertson (University of Illinois) to whom the author is extremely grateful. Additional collaborations with Dr. Mike Jenkins (University of Oxford) are also greatly appreciated. All of this is based on the excellent work of students and post docs, Keith Tappin, John Vetrano, Mike Bench, Tom Black, Kes Smalinskas and Tho Bui. Recent collaboration with Dr. Hiroshi Fukushima (Hiroshima University) is warmly acknowledged.

\section{REFERENCES}

1. M. L. Jenkins, M. A. Kirk and W. J. Phythian, J. Nucl. Mater. 205, 16 (1993).

2. M. Ghaly and R. S. Averback, Phys. Rev. Lett. 72, 364 (1994).

3. T. Diaz de la Rubia and W. J. Phythian, J. Nucl. Mater. 191-194, 108 (1992).

4. I. M. Robertson, J. S. Vetrano, M. A. Kirk and M. L. Jenkins, Phil. Mag. A 63, 299 (1991).

5. M. A. Kirk and H. W. Weber, in Studies of High Temperature Superconductors Volume 10, edited by A. Narlikar (Nova Science Publishers, Commack, NY, 1993) p. 254.

6. M. A. Kirk and L. R. Greenwood, J. Nucl. Mater. 80, 159 (1979).

7. T. X. Bui, E. Sirois, I. M. Robertson and M. A. Kirk, in Effects of Radiation on Materials, Eds: A. S. Kumar, R.E. Stoller and D.S. Gelles, (ASTM, 1991)STP1125,463 .

8. M. A. Kirk, I. M. Robertson, M. L. Jenkins, C. A. English, T. J. Black and J. S. Vetrano, J. Nucl. Mater. 149, 21 (1987).

9. I. M. Robertson, M. A. Kirk and W. E. King, Scripta Met. 18, 317 (1984).

10. J. S. Vetrano, I. M. Robertson and M. A. Kirk, Scripta Met. 24, 157 (1990).

11. Y. Shimomura, M. W. Guinan, H. Fukushima, P. A. Hahn and M. Kiritani, J. Nucl. Mater. 155-157, 1181 (1988).

12. J. M. Muncie, B. L. Eyre and C. A. English, Phil. Mag. A, 52, 309 (1985).

13. B. W. O. Shepherd, M. L. Jenkins and C. A. English, Phil. Mag. A, 56, 458 (1987).

14. M. A. Kirk, M. L. Jenkins and H. Fukushima, to be published, (1995).

15. C. A. English, A. J. E. Forman, W. J. Phythian, D. J. Bacon and M. L. Jenkins, Mat. Sci. Forum, 97-99, 1 (1992).

16. S. Mueller, M. L. Jenkins and C. Abromite, to be published, (1995).

17. T. J. Black, M. L. Jenkins, C. A. English and M. A. Kirk, Proc. R. Soc. Lond. A 409, $177(1987)$.

18. K. B. Winterbon, P. Sigmund and J. B. Sanders, K. danske Vidensk. Selsk. Skr, 37 (40), (1970).

19. J. S. Vetrano, I. M. Robertson and M. A. Kirk, Phil. Mag. A, 68, 381 (1993).

20. I. M. Robertson, D. K. Tappin and M. A. Kirk, Phil. Mag. A, 68, 843 (1993).

21. D. K. Tappin, I. M. Robertson and M. A. Kirk, Phil. Mag A, 70, 463 (1994).

22. C. P. Flynn and R. S. Averback, Phys. Rev. B 38, 7118 (1988).

23. A. Y. Stathopoulos, C. A. English, B. L. Eyre and P. B. Hirsch, Phil. Mag. A 44, 309 (1981). 